Portland State University

PDXScholar

7-2019

\title{
Divergent Trends in Life Expectancy Across the Rural-Urban Gradient and Association with Specific Racial Proportions in the Contiguous USA 2000-2005
}

\author{
Yun Jian \\ University of Alabama at Birmingham \\ Lucas Neas \\ U.S. Environmental Protection Agency \\ Lynne C. Messer \\ Portland State University, lymesser@pdx.edu \\ Christine L. Gray \\ University of North Carolina \\ Jyotsna S. Jagai \\ University of Illinois
}

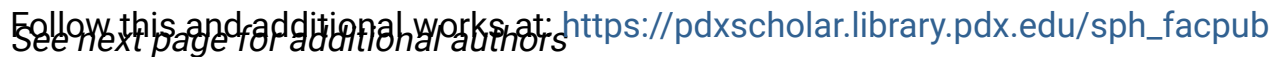

Part of the Public Health Commons

Let us know how access to this document benefits you.

\begin{abstract}
Citation Details
Jian, Y., Neas, L., Messer, L. C., Gray, C. L., Jagai, J. S., Rappazzo, K. M., \& Lobdell, D. T. (2019). Divergent trends in life expectancy across the rural-urban gradient and association with specific racial proportions in the contiguous USA 2000-2005. International Journal Of Public Health.
\end{abstract}

This Article is brought to you for free and open access. It has been accepted for inclusion in OHSU-PSU School of Public Health Faculty Publications and Presentations by an authorized administrator of PDXScholar. Please contact us if we can make this document more accessible: pdxscholar@pdx.edu. 
Authors

Yun Jian, Lucas Neas, Lynne C. Messer, Christine L. Gray, Jyotsna S. Jagai, Kristen M. Rappazzo, and Danelle T. Lobdell 


\title{
Divergent trends in life expectancy across the rural-urban gradient and association with specific racial proportions in the contiguous USA 2000-2005
}

\author{
Yun Jian ${ }^{1,2} \cdot$ Lucas Neas $^{3} \cdot$ Lynne C. Messer $^{4} \cdot$ Christine L. Gray $^{2,5} \cdot$ Jyotsna S. Jagai $^{6} \cdot$ Kristen M. Rappazzo $^{3} \cdot$ \\ Danelle T. Lobdell ${ }^{3}$
}

Received: 21 December 2017 / Revised: 17 December 2018/Accepted: 19 June 2019

(C) This is a U.S. Government work and not under copyright protection in the US; foreign copyright protection may apply 2019

\begin{abstract}
Objectives To estimate county-level adult life expectancy for Whites, Black/African Americans (Black), American Indian/ Alaska Native (AIAN) and Asian/Pacific Islander (Asian) populations and assess the difference across racial groups in the relationship among life expectancy, rurality and specific race proportion.

Methods We used individual-level death data to estimate county-level life expectancy at age 25 (e25) for Whites, Black, AIAN and Asian in the contiguous USA for 2000-2005. Race-sex-stratified models were used to examine the associations among e25, rurality and specific race proportion, adjusted for socioeconomic variables.

Results Lower e25 was found in the central USA for AIANs and in the west coast for Asians. We found higher e25 in the most rural areas for Whites but in the most urban areas for AIAN and Asians. The associations between specific race proportion and e25 were positive or null for Whites but were negative for Blacks, AIAN, and Asians. The relationship between specific race proportion and e25 varied across rurality.

Conclusions Identifying differences in adult life expectancy, both across and within racial groups, provides new insights into the geographic determinants of life expectancy disparities.
\end{abstract}

Keywords Life expectancy at age $25 \cdot$ Rurality $\cdot$ American Indian/Alaska Native population - Asian/Pacific Islander population $\cdot$ Specific race proportion $\cdot$ Contiguous USA

\section{Introduction}

Large disparities in life expectancy in the USA have been observed across races and geographic areas. Between races in the USA, Black and Asian populations demonstrated the largest difference in life expectancy at birth $(\mathrm{e} 0)$, which

Disclaimer: The views expressed in this manuscript are those of the authors and do not necessarily reflect the views or policies of the US EPA. Mention of trade names or commercial products does not constitute endorsement or recommendation for use.

Electronic supplementary material The online version of this article (https://doi.org/10.1007/s00038-019-01274-5) contains supplementary material, which is available to authorized users.

Danelle T. Lobdell

lobdell.danelle@epa.gov

Extended author information available on the last page of the article was about 12 years in 2009 (Henry J Kaiser Family Foundation 2009). The difference in $\mathrm{e} 0$ was about 5 years between Black and White populations (Levine et al. 2001; National Center for Health Statistics 2016; Crimmins and Saito 2001; Harper et al. 2007). Across geographies, county-level differences in e0 between the best-off and worst-off counties were about 18 years for Black males, 14 years for Black females, 15 years for White males, and 11 years for White females in 2001 (Murray et al. 2006).

Although county-level life expectancy for Whites and Black/African Americans (Black) has been reported, very little is known for American Indian/Alaska Native (AIAN) and Asian/Pacific Islanders (Asian). Studies of life expectancy usually pool AIAN and Asians at the national/regional levels (Crimmins and Saito 2001; Murray et al. 2006), or pool them by socioeconomic status (Singh and Siahpush 2006, 2014), or exclude them altogether. However, the determinants of life expectancy for Asian and/or AIAN populations may differ from Whites and Blacks and 
may also vary geographically. Life expectancy estimates at a geographic unit other than the national level (e.g., county level) for AIANs and Asians would enable a better understanding of their health status and potential disparities in these racial groups that have been under-studied.

Individual factors, such as income and education, are linked with variations in life expectancy (Singh and Siahpush 2006; Chetty et al. 2016). However, less is understood about area-level measures (such as rurality) that may represent differential environmental exposures and thus potentially influence life expectancy. Limited studies in the USA have explored the differences in life expectancy across the rural-urban gradient. Singh and Siahpush (2014) found higher $\mathrm{e} 0$ in metropolitan areas compared to nonmetropolitan areas. However, Geronimus et al. (2001) found life expectancy at age 16 (e16) was higher in rural areas for Black and Whites. Besides contrasting results, the causes of the rural-urban disparities remain unclear, motivating further investigations into life expectancy across the rural-urban gradient.

Another area-level variable potentially influencing life expectancy is the proportion of a given race residing in a neighborhood, which we will refer to as the specific race proportion. This race proportion matters for understanding the geographic determinants of mortality because while mortality rates differ by race, so do the factors that may contribute to mortality differentials, such as investment in neighborhood resources and health promotion infrastructure (Jackson et al. 2000; Inagami et al. 2006). A few studies have reported proportions of the same race were linked with race-specific mortality, but the results were inconsistent (Jackson et al. 2000; Inagami et al. 2006; Fang et al. 1998; Hutchinson et al. 2009). The relationship between specific race proportion and life expectancy has rarely been assessed previously and needs further investigation.

In this study, we addressed these questions by (1) estimating county-level life expectancy at age 25 (e25) in the contiguous USA for Whites, Blacks, AIAN, and Asians, and investigating the geographic patterns in e25; (2) assessing the differences in life expectancy across rurality [defined by Rural-Urban Continuum Codes (United States Department of Agriculture 2016)]; (3) assessing the associations between life expectancy and county-level-specific race proportion (county-level proportion of a given race, same race\%); and (4) examining potential interactions between rurality and specific race proportion for Whites and Blacks.

\section{Methods}

Data for mortality, population, rurality, and other sociodemographic variables

Individual death data from the National Center for Health Statistics were aggregated as death counts into 5-year age groups by county and race-sex groups for the contiguous USA for years 2000-2005 (National Center for Health Statistics 2000-2005). We focused on the contiguous USA in this study because of the small number of deaths in Alaska and Hawaii and because of the county changes in Alaska that prevented matching county-level mortality and population data during the study period.

We used bridged-race population estimates to calculate 5 -year mortality rates. The bridged population data mapped 31 race categories, as specified in the 1997 Office of Management and Budget standards for the collection of data on race and ethnicity, to the four race categories specified under the 1977 standards (the same as race categories in mortality registration) (Ingram et al. 2003). Agespecific bridged population estimates for 2000-2005 were obtained from National Cancer Institute's Surveillance, Epidemiology, and End Results Program (National Cancer Institute's Surveillance Epidemiology and End Results 2016).

The urban-rural gradient was represented by the 2003 Rural Urban Continuum Codes (RUCC), which distinguished metropolitan counties by population size, and nonmetropolitan counties by degree of urbanization and adjacency to a metro area (United States Department of Agriculture 2016). The nine RUCC groups were condensed into four groups as has been done elsewhere: metropolitan urbanized (RUCC 1, urban population $\geq 250,000$, categories 1-3 in the original nine classification), nonmetropolitan urbanized (RUCC 2, urban population of 20,000-250,000, categories 4 and 5 in the original classification), less urbanized (RUCC 3, urban population of 2500-19,999, categories 6 and 7 in the original classification), and thinly populated (RUCC 4, completely rural, < 2500 urban population, categories 8 and 9 in the original classification) areas (Luben et al. 2009; Messer et al. 2010).

We obtained county-level sociodemographic data for 2000-2005 from the US Census Bureau. These included median household income, percent of population attaining greater than high school education (high school\%), and percent of county occupied rental units (rent\%). We obtained county violent crime from Uniform Crime Reports and used it to calculate mean number of violent crimes per capita (Federal Bureau of Investigation 2010). These four variables were used for confounder control in adjusted models. 


\section{Estimation of life expectancy at age 25}

In our study, we focused on e25 to represent adult life expectancy. County-level e25 was calculated using agespecific death rates for every 5 -year group starting at age 25 . The observed death rates (death counts/population) can be unstable for counties with small populations, which leads to inaccuracy in the estimated life expectancy. Therefore, we used a random slope, random intercept Poisson regression to stabilize the county-level and racesex-specific death rates. We constructed a log-linear model between death rate and age to borrow strength across age groups when estimating age-specific death rate. The loglinear relationship between death rate and age has been used in other life expectancy work (Chetty et al. 2016) and was observed for our mortality data for age between 25 and 84. We estimated e25 for eight race-sex groups (White, Black, AIAN, Asian, male and females). Ethnicity (Hispanics vs non-Hispanic) was not estimable due to data limitation. To address unstable estimates in many counties for ages over 84 , we linearly extrapolated, through age $100-104$, the logs of the 5-year age-specific mortality rates for ages $25-84$ by sex and race. The details of the model for estimating e25 can be found in Electronic Supplementary Materials (ESM). We reported e25 for each sexrace group using median and interquartile range since mortality is not normally distributed.

\section{Statistical analysis}

We used linear regression models to assess the relationships among county-level e25, rurality, and specific race proportion: (1) models with only RUCC as the independent variable; (2) models with only specific race proportion as the independent variable (3) models with both specific race proportion and RUCC, adjusted for sociodemographic variables (household income, school\%, rent $\%$, and number of violent crimes per capita); and (4) models with RUCC, specific race proportion, and the interaction between RUCC and specific race proportion, also adjusted for sociodemographic variables. Specific race proportion was represented by county-level proportion of a given race (same race\%, for example, White\% for White population and Black\% for Black population). The first three models were run for all the eight race-sex groups and the last model was run only for Whites and Blacks to test our hypothesis that the associations of e 25 and specific race proportion may vary across the rural-urban gradient. All models were run separately for the race-sex groups. For the rural-urban gradient, life expectancy in RUCC 2 (nonmetro urbanized), RUCC 3 (less populated), and RUCC 4 (thinly populated) was compared against RUCC 1 (metropolitan areas). We assessed models both with and without the adjusting sociodemographic variables and compared their results to check the influence of these variables on the associations among e25, rurality, and specific race proportion; we reported estimates for the adjusted models. Specific race proportion and sociodemographic variables were standardized (mean $=0$ and standard deviation $=1$ ) prior to inclusion in the model to ensure the comparability among the model coefficients (Table ESM1). Thus, the results were presented as the change in life expectancy per one standard deviation change in specific race proportion.

All models were assessed for violations of model assumptions. Counties with fewer than 12 deaths (2 deaths per year per county on average) during 2000-2005 for the sex-race group were excluded (Table ESM2 shows the remaining number of counties for each race-sex group). For sensitivity analyses, we also tested models in which counties with fewer than 72 deaths (6 deaths per year per county on average) during 2000-2005 were excluded, and the results (not shown) were similar. For Asian populations in RUCC 3 (less populated) and 4 (thinly populated), there were fewer than 15 counties with estimated e25. Thus, they were not included in the analyses.

\section{Results}

\section{Estimated life expectancy at age 25}

Overall, in the contiguous USA, the estimated county-level e25 (mean remaining years of life at age 25) was highest for Asian females: 62.6 years $(61.1,64.3)$ (median and interquartile range) and lowest for Black males 46.7 years (45.1, 48.8) (Table ESM2). The AIANs had the largest variations in e25 (for both males and females) compared to other race-sex groups (Table ESM2). Geographically, lower e 25 for the Whites and Blacks were observed in the southeast (Fig. 1). Lower e25 for AIANs was found in the central part of USA, and lower e25 for Asians was observed in the western coast for the metropolitan and nonmetro urbanized counties (Fig. 1).

\section{Difference of life expectancy at age 25 across urban-rural status}

The model with only RUCC showed that for Whites the highest e25 was found in thinly populated areas (RUCC 4), followed by metropolitan areas (RUCC 1). However, for Blacks, AIANs, and Asians, the highest e25 was found in metropolitan areas (RUCC 1) (Table ESM3). The model with RUCC and specific race proportion adjusted for sociodemographic variables showed a different pattern for 

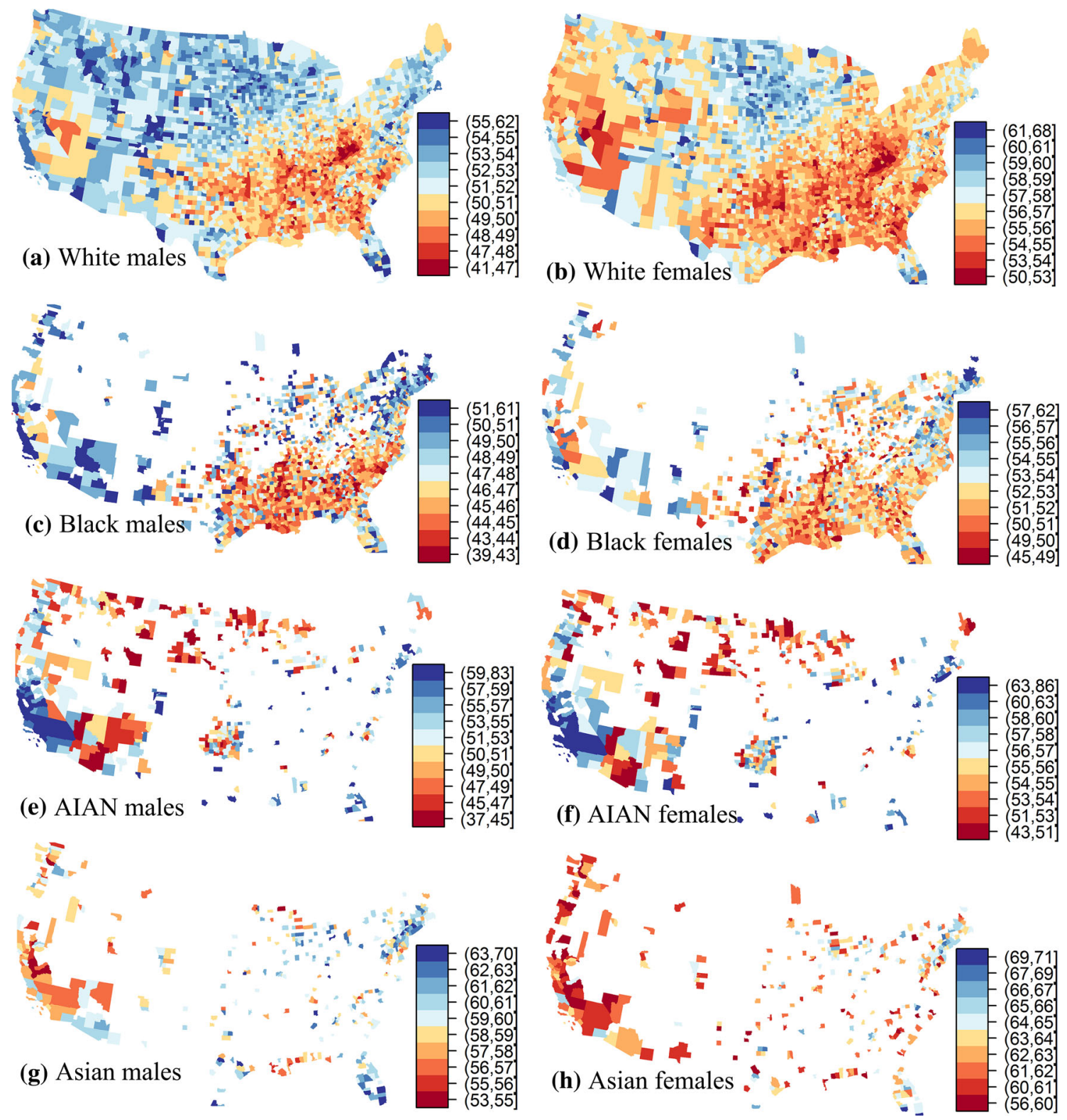

Fig. 1 County-level life expectancy at age 25 for the contiguous USA 2000-2005 (Deciles differed among sex-race groups). White patches: counties with no estimated life expectancy

the trend of e 25 across rurality (Table ESM4). For Whites, we observed a monotonically increasing trend for e25 from the most urban (RUCC 1) to the most rural areas (RUCC 4). For Blacks, we found that the highest e 25 was found in thinly populated areas (RUCC 4). The results for AIAN were similarly best in RUCC 4 .

\section{Difference of life expectancy at age $\mathbf{2 5}$ across specific race proportion}

The model with only specific race proportion resulted in positive associations between e 25 and White $\%$ for White, but mostly negative associations between e 25 and specific race proportion for other groups (Fig. 2 and Table ESM5). The negative associations suggested that e 25 was lower in counties with larger proportion of the same race. For example, a one standard deviation increase (6.7 percentage points) in AIAN\% was associated with $-1.2(-1.4$, - 1.0) (mean and 95\% CI) and - $0.8(-1.0,-0.6)$ years change in e25 of AIAN males and females, respectively. The model with both RUCC and specific race proportion had similar results with the model with only specific race proportion (Fig. 2).

The model with RUCC, specific race proportion, and adjusting sociodemographic variables also showed mostly negative associations between e25 and specific race 

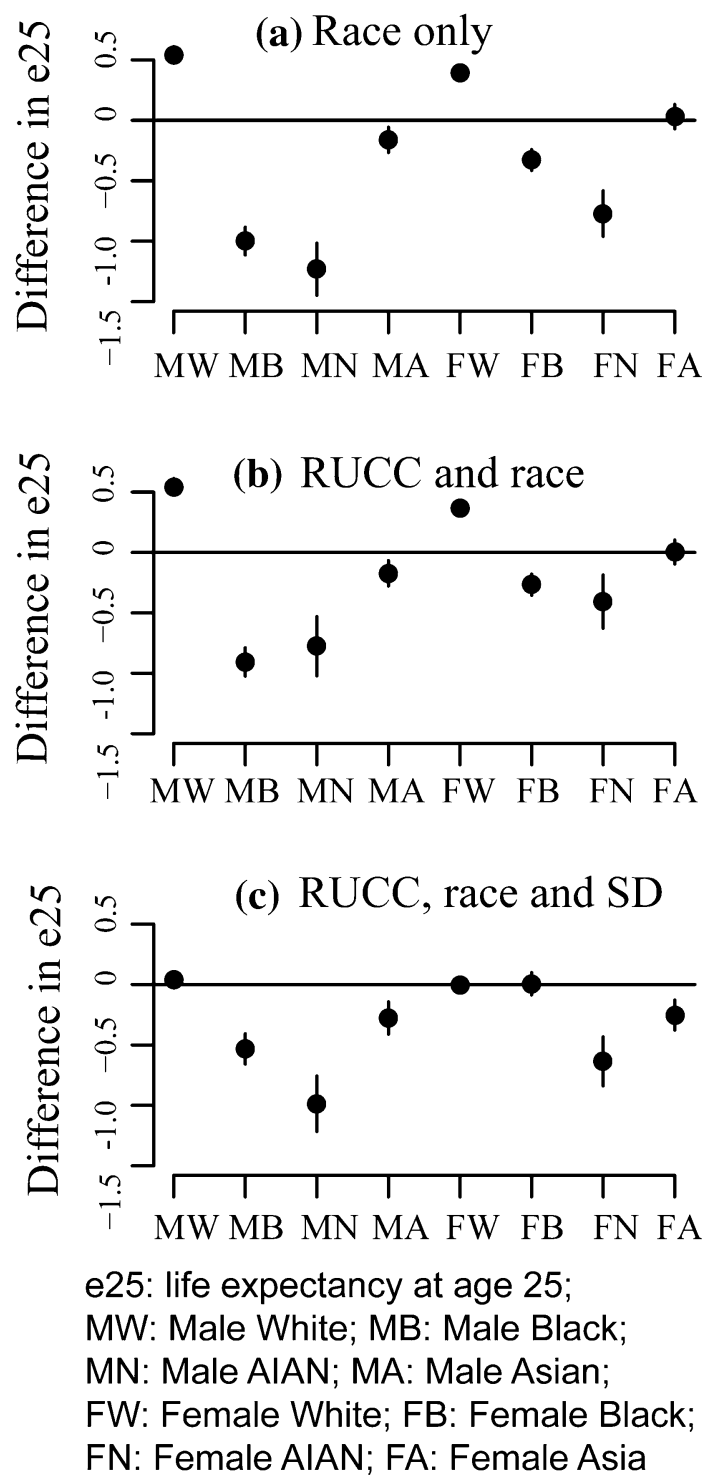

Fig. 2 Differences in life expectancy at age 25 (e25) per one standard deviation change in $(95 \%$ Confidence Interval) specific race proportion from the models for the contiguous USA 2000-2005 with a specific race proportion only, $\mathbf{b}$ the models with both Rural Urban Continuum Code (RUCC) and specific race proportion, and $\mathbf{c}$ the models with RUCC, specific race proportion, and adjusting sociodemographic variables $(\mathrm{SD})$

proportion for Black, AIAN, and Asian populations. However, the associations between e25 and specific race proportion, adjusted for sociodemographic variables, were null for Whites (Fig. 2 and Table ESM4).

\section{Interactions between rurality and specific race proportion}

In the models with interactions between rurality and specific race proportion, adjusted for sociodemographic variables, some statistically significant interactions were observed for both Blacks and Whites (Table ESM6). However, the directionality of the association varied between races, and the net effects were generally more negative for Blacks, particularly in RUCC 1 (metropolitan) and RUCC 2 (non-metro urbanized) (Table ESM6, Fig. 3). For White males, the association between e 25 and White $\%$ was significantly positive in the metropolitan areas $(0.2$ $(0.1,0.3))$, but significantly negative in thinly populated areas $(-0.1(-0.2,0.0))$. For Black females, the association between e25 and Black\% was significantly negative in the metropolitan areas $(-0.1(-0.3,0.0))$, but significantly positive in thinly populated areas $(0.3(0.0,0.5))$. For White females, the e25-racial share association was null in all RUCCs. For Black males, the association was significantly negative in all RUCCs except the thinly populated areas.

\section{Discussion}

In this study, we estimated county-level life expectancy at age 25 (e25) for eight race-sex groups in the contiguous USA and modeled the associations among e25, rurality and specific race proportion. We found different trends in e25 across the rural-urban gradient and different relationships between e25 and specific race proportion among Whites, Blacks, AIANs, and Asians. We also observed significant interactions between specific race proportion and rurality, suggesting varying relationships between e25 and specific race proportion in rural and urban areas for Whites and Blacks.

County-level life expectancy for AIANs and Asians has rarely been reported. Our analyses showed, for the first time to our knowledge, the geographic patterns of e 25 for AIAN in the contiguous USA and Asian populations in metropolitan and non-metro urbanized counties. The difference in the geographic pattern may suggest that the driving factors for life expectancy vary spatially within racial groups.

Wide disparities in the county-level e25 were observed within-race-sex groups. For example, the interquartile range was 48.7-55.6 for AIAN males and was 53.5-59.3 for AIAN females. The magnitude of the within-race difference in e25 was comparable to the between-race difference for both e25 in our study and life expectancy at birth (e0) from previous studies (Harper et al. 2007; Murray et al. 2006). The wide gaps in the county-level e25 suggested large disparities in overall health status within a racial group across geographic locations.

Our results suggest the trend of e 25 across rural-urban gradient was different between-race groups. Our results for the White and Black populations when adjusting for sociodemographic variables were consistent with the 
Fig. 3 Difference in life expectancy at age 25 (e25) per one standard deviation change (95\% Confidence Interval) in specific race proportion across rurality from the interaction models with adjusting variables for the contiguous USA 20002005. RUCC Rural Urban Continuum Code; RUCC 1 metropolitan areas; RUCC 2 non-metro urbanized areas; RUCC 3 areas less populated; RUCC 4 thinly populated areas
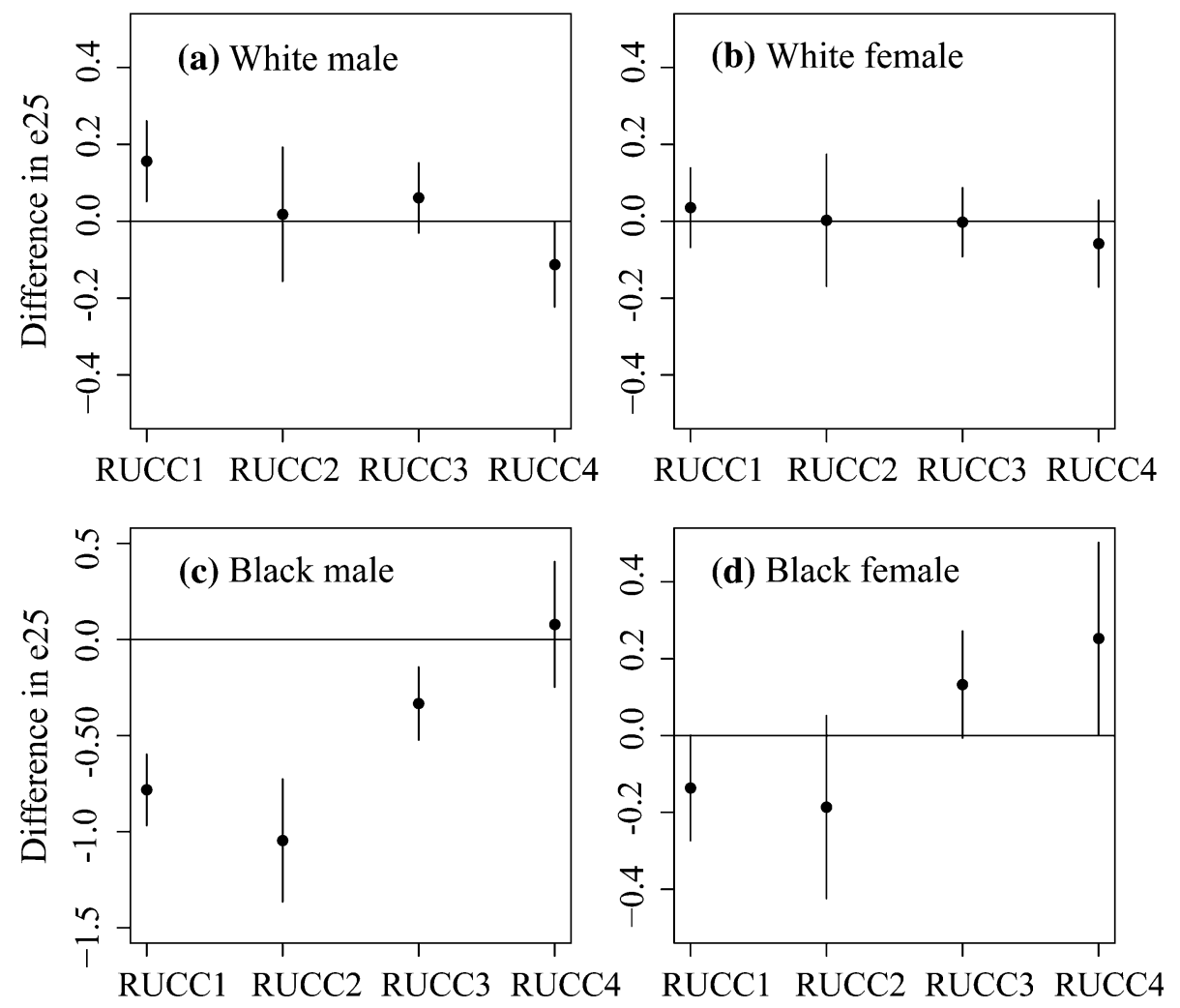

findings of Geronimus et al. (2001) which showed that rural residents outlive urban residents. Unadjusted results (not shown) had been similar to the results of Singh and Siahpuch (2014) for Black population, which found higher life expectancy at birth (e0) in metropolitan areas. However, we observed the highest e25 in the most rural areas for White population, which was different from the results of Singh and Siahpuch (2014). Of note, our study estimated e25 while the study of Geronimus et al. (2001) focused on e16, and Singh and Siahpuch (2014) reported e0. This suggests the trends of overall health status across ruralurban gradient may differ among age groups.

This study also reported the associations between specific race proportion (same race\%) and e25 in the contiguous USA and how they varied across rurality. The negative associations between specific race proportion and e25 for Black, AIAN, and Asian populations were consistent with previous studies which showed worse health outcomes in areas with smaller percent of White population (Jackson et al. 2000; Mellor and Milyo 2004; Hart et al. 1998). However, our models with the interaction between specific race proportion and rurality further showed the magnitude and the signs of associations between specific race proportion and e 25 were different in the most urban and the most rural areas for White and Black populations.

In our analyses, we adjusted for sociodemographic variables. Compared to unadjusted models, for rurality, these models showed different trends of e25 across rural- urban status for White and Black populations; for specific race proportion, these models had similar results for Black, AIAN, and Asian populations, but different results for White populations. The differences between adjusted and unadjusted models suggest that part of the differences in e25 across rurality and across specific race proportion gradient may be attributed to the sociodemographic variables. Both the adjusted and unadjusted models may misattribute the relationships because the true causal pathways of rural-urban status and specific race proportion on adult life expectancy are unknown. It is possible that including the sociodemographic variables in the model may remove some of the legitimate differences in e 25 that are attributable to rurality and specific race proportion. However, we were more concerned that models without sociodemographic adjustment may misattribute the observed associations to rurality or specific racial proportion. For example, if education influences life expectancy and it is also determined by other unmeasured factors (such as regional differences), leaving it unadjusted can result in misleading associations. Therefore, we presented adjusted models.

In most previous studies, life expectancy was estimated using either observed age-specific death rates or agespecific death estimated separately for age groups (Murray et al. 2006; Kulkarni et al. 2011; Dominici et al. 2015). In our study, we assumed a log-linear relationship between death rate and age starting at 25 to pool information across 
age groups. Additionally, the random intercept and random slope model also allowed us to borrow strength across race, sex and county groups. This method likely produced more robust estimates of life expectancy for counties with small populations and enabled us to estimate county-level e25 for AIANs and Asians which could not be calculated previously.

Our inability to account for individual-level SES is a limitation of this study. Further, macro-level characteristics, such as social security benefits or public welfare systems, may contribute to differential mortality. This study also lacked detailed information about differential migration of individuals. Rural areas may appear healthier as the infirm and disabled may prefer to stay in more urbanized areas with more social services. It is also possible that people who migrate to urban areas for job opportunities are healthier than the average population (Diaz et al. 2016; Schenker et al. 2014). If this is the case, the apparent difference in e 25 across urban/rural status may be affected by migration patterns. Further studies comparing life expectancy between the migrating population and non-migrating populations may shed light on this problem.

Another limitation is our inability to address ethnicity (Hispanic vs Non-Hispanic) separately in our analyses. The Hispanic population was estimated to have longer life expectancy than Non-Hispanic White and Non-Hispanic Black populations at the national level (National Center for Health Statistics 2016). However, little is known about their life expectancy at the county level. We could not separate ethnicity because it was not available in the bridged-race population estimates. Future studies about county-level life expectancy for Hispanic and Non-Hispanic populations will be valuable for understanding their overall health status.

The estimation of e 25 in this study relied on the validity of the population and death data used. Misclassifications of race in death certificates have been reported, and the influence of this on the estimated e25 could differ by racial groups (Casper et al. 2003; Rosenberg et al. 1999). The e25 of Asians and AIANs are more likely to be impacted by misclassification compared to Whites and Black due to their smaller population (Casper et al. 2003; Rosenberg et al. 1999). This difference in the racial misclassification may also affect our estimated associations between e25, rurality, and specific race proportion.

\section{Conclusions}

This study was the first to assess county-level adult life expectancy (e25) for Asians and AIANs, and further highlighted racial heterogeneity in e25 by geographic region, rurality, and specific race proportion. Asians and
AIANs had different geographic patterns in e25 and different associations between rurality status and e25, when compared to Whites and Blacks. Specific race proportion was associated with lower e25 for Blacks, AIANs, and Asians, but higher e25 for Whites. Significant interactions were observed between rurality and specific race proportion for Whites and Blacks, suggesting that the relationship between specific race proportion (same race\%) and adult life expectancy varies across rural-urban gradient. The results of this study revealed the wide difference in adult life expectancy both within and between racial groups, providing new insights into the geographic determinants of life expectancy disparities.

Acknowledgement The US Environmental Protection Agency (EPA) Office of Research and Development (ORD) partially funded the research with L.C.M. (contracts EP12D000264, EP09D000003 and EP17D000079), J.S.J (contract EP17D000063), Y.J., J.S.J., and C.L.G. were supported in part by an appointment to the Internship/ Research Participation Program at Office of Research and Development (NHEERL), US EPA, administered by the Oak Ridge Institute for Science and Education through an interagency agreement between the US Department of Energy and the US EPA. We also thank Bryan Hubbell, Patricia Murphy, and Stephanie DeFlorio-Barker for their insightful suggestions to this study.

\section{Compliance with ethical standards}

Ethical approval We used mortality data to calculate life expectancy in this study. Thus, by definition, it is non-human subject research. No human subject ethics approval is needed as determined by the Human Research Protocol Officer from the National Health and Environmental Effects Research Laboratory, United States Environmental Protection Agency.

Conflict of interest The authors declare that they have no conflict of interest.

\section{References}

Casper ML, Barnett E, Williams GI Jr, Halverson JA, Braham VE, Greenlund KJ (2003) Atlas of stroke mortality: racial, ethnic, and geographic disparities in the United States. Department of Health and Human Services, Centers for Disease Control and Prevention, Atlanta

Chetty R, Stepner M, Abraham S et al (2016) The association between income and life expectancy in the united states, 2001-2014. JAMA 315(16):1750-1766. https://doi.org/10. 1001/jama.2016.4226

Crimmins EM, Saito Y (2001) Trends in healthy life expectancy in the United States, 1970-1990: gender, racial, and educational differences. Soc Sci Med 52(11):1629-1641. https://doi.org/10. 1016/S0277-9536(00)00273-2

Diaz CJ, Koning SM, Martinez-Donate AP (2016) Moving beyond Salmon bias: mexican return migration and health selection. Demography 53(6):2005-2030. https://doi.org/10.1007/s13524016-0526-2

Dominici F, Wang Y, Correia AW, Ezzati M, Pope CA, Dockery DW (2015) Chemical composition of fine particulate matter and life 
expectancy: in 95 US counties between 2002 and 2007. Epidemiology (Cambridge, Mass) 26(4):556-564. https://doi. org/10.1097/EDE.0000000000000297

Fang J, Madhavan S, Bosworth W, Alderman MH (1998) Residential segregation and mortality in New York City. Soc Sci Med 47(4):469-476. https://doi.org/10.1016/S0277-9536(98)00128-2

Federal Bureau of Investigation (2010) Uniform crime reporting. https://ucr.fbi.gov/. Accessed 26 Aug 2010

Geronimus AT, Bound J, Waidmann TA, Colen CG, Steffick D (2001) Inequality in life expectancy, functional status, and active life expectancy across selected black and white populations in the United States. Demography 38(2):227-251

Harper S, Lynch J, Burris S, Davey Smith G (2007) Trends in the black-white life expectancy gap in the united states, 1983-2003. JAMA 297(11):1224-1232. https://doi.org/10.1001/jama.297.11. 1224

Hart KD, Kunitz SJ, Sell RR, Mukamel DB (1998) Metropolitan governance, residential segregation, and mortality among African Americans. Am J Public Health 88(3):434-438

Henry J Kaiser Family Foundation (2009). Life expectancy at birth (in years). http://kff.org/other/state-indicator/life-expectancy-by-re/ ?currentTimeframe $=0 \&$ sortModel $=\% 7 \mathrm{~B} \% 22$ colId $\% 22: \% 22 \mathrm{Loca}$ tion\%22,\%22sort\%22:\%22asc\%22\%7D

Hutchinson RN, Putt MA, Dean LT, Long JA, Montagnet CA, Armstrong K (2009) Neighborhood racial composition, social capital and black all-cause mortality in Philadelphia. Soc Sci Med 68(10):1859-1865. https://doi.org/10.1016/j.socscimed. 2009.02.005

Inagami S, Borrell LN, Wong MD, Fang J, Shapiro MF, Asch SM (2006) Residential segregation and Latino, black and white mortality in New York City. J Urban Health: Bull NY Acad Med 83(3):406-420. https://doi.org/10.1007/s11524-006-9035-8

Ingram DD, Parker JD, Schenker N, Weed JA, Hamilton B, Arias E, et al (2003) United States Census 2000 population with bridged race categories. National Center for Health Statistics. Vital Health Stat 2(135). http://www.cdc.gov/nchs/data/series/sr_02/ sr02_135.pdf

Jackson SA, Anderson RT, Johnson NJ, Sorlie PD (2000) The relation of residential segregation to all-cause mortality: a study in black and white. Am J Public Health 90(4):615-617

Kulkarni SC, Levin-Rector A, Ezzati M, Murray CJ (2011) Falling behind: life expectancy in US counties from 2000 to 2007 in an international context. Popul Health Metr 9(1):16. https://doi.org/ 10.1186/1478-7954-9-16

Levine RS, Foster JE, Fullilove RE, Fullilove MT, Briggs NC, Hull $\mathrm{PC}$ et al (2001) Black-white inequalities in mortality and life expectancy, 1933-1999: implications for healthy people 2010. Public Health Rep 116(5):474-483. https://doi.org/10.1093/phr/ 116.5.474

Luben TJ, Messer LC, Mendola P, Carozza SE, Horel SA, Langlois PH (2009) Urban-rural residence and the occurrence of neural tube defects in Texas, 1999-2003. Health Place 15(3):863-869. https://doi.org/10.1016/j.healthplace.2009.02.006

Mellor JM, Milyo JD (2004) Individual health status and racial minority concentration in US states and counties. Am J Public Health 94(6):1043-1048

Messer LC, Luben TJ, Mendola P, Carozza SE, Horel SA, Langlois PH (2010) Urban-rural residence and the occurrence of cleft lip and cleft palate in Texas, 1999-2003. Ann Epidemiol 20(1):32-39. https://doi.org/10.1016/j.annepidem.2009.09.006

Murray CJ, Kulkarni SC, Michaud C, Tomijima N, Bulzacchelli MT, Iandiorio TJ et al (2006) Eight Americas: investigating mortality disparities across races, counties, and race-counties in the United States. PLoS Med 3(9):e260. https://doi.org/10.1371/journal. pmed.0030260

National Cancer Institute's Surveillance Epidemiology and End Results (2016) U.S. Population data-1969-2014. http://seer. cancer.gov/popdata/

National Center for Health Statistics (2000-2005)

National Center for Health Statistics (2016) Health, United States, 2015: with special feature on racial and ethnic health disparities. Hyattsville, MD

Rosenberg HM, Maurer JD, Sorlie PD, Johnson NJ (1999) Quality of death rates by race and Hispanic origin: a summary of current research, 1999. National Center for Health Statistics. Vital Health Stat 2(128):1999

Schenker MB, Castañeda X, Rodriguez-Lainz A (2014) Migration and health: a research methods handbook. University of California Press, Berkeley

Singh GK, Siahpush M (2006) Widening socioeconomic inequalities in US life expectancy, 1980-2000. Int $\mathrm{J}$ Epidemiol 35(4):969-979. https://doi.org/10.1093/ije/dy1083

Singh GK, Siahpush M (2014) Widening rural-urban disparities in life expectancy, US., 1969-2009. Am J Prev Med 46(2):e19e29. https://doi.org/10.1016/j.amepre.2013.10.017

United States Department of Agriculture (2016) Rural-urban continuum codes. http://www.ers.usda.gov/data-products/rural-urbancontinuum-codes.aspx

Publisher's Note Springer Nature remains neutral with regard to jurisdictional claims in published maps and institutional affiliations.

\section{Affiliations}

\section{Yun Jian ${ }^{1,2} \cdot$ Lucas Neas $^{3} \cdot$ Lynne C. Messer ${ }^{4}$ Christine L. Gray ${ }^{2,5} \cdot$ Jyotsna S. Jagai $^{6} \cdot$ Kristen M. Rappazzo $^{3}$. Danelle T. Lobdell ${ }^{3}$}

1 Informatics Institute, University of Alabama at Birmingham, Birmingham, USA

2 National Health and Environmental Effects Research Laboratory, Oak Ridge Institute for Science and Education at the U.S. Environmental Protection Agency, Chapel Hill, NC, USA

3 National Health and Environmental Effects Research Laboratory, U.S. Environmental Protection Agency (USEPA), MD 58A, Research Triangle Park, NC 27711, USA
4 School of Community Health, College of Urban and Public Affairs, Portland State University, Portland, OR, USA

5 Gillings School of Global Public Health, University of North Carolina, Chapel Hill, NC, USA

6 School of Public Health, Division of Environmental and Occupational Health Sciences, University of Illinois, Chicago, Chicago, IL, USA 